\title{
Capacité de prise de décision des femmes en matière de santé de la reproduction, et la capacité d'autonomisation par l'augmentation des revenus à travers des activités de maraichage dans le cercle de Kangaba au Mali
}

\section{Women's decision-making capacity in reproductive health and the capacity to empower themselves by increasing income through gardening activities in the Kangaba circle in Mali}

\author{
Diarra $\mathrm{NH}^{1}$, Ag lknane $\mathrm{A}^{2}$, Diop $\mathrm{S}^{3}$.
}

\begin{abstract}
1 Médecin en Santé Publique, Faculté de Médecine, Université des Sciences, des Techniques et Technologies de Bamako (Mali). 2 Professeur en Santé Publique-Nutrition, Faculté de Pharmacie, USTTB, Bamako (Mali)

${ }^{3}$ Professeur en Anthropologie Médicale, Bioéthique, Faculté de Médecine, Université des Sciences, des Techniques et Technologies de Bamako (Mali)
\end{abstract}

RESUME : Les femmes économiquement autonomes sont plus engagées dans leur propre santé aussi bien que dans celle de leurs enfants. Dans cette optique, I'ONG IAMANEH Suisse, en collaboration avec I'ONG GAD, a mis en œuvre un projet d'autonomisation des femmes dans le cercle de Kangaba. Nous avons mené cette étude trois ans après le début du projet pour évaluer l'impact de ce projet. Elle a été réalisée en août 2018 dans les communes de Balan Bakama, Séléfougou et Maramandougou du cercle de Kangaba. Une étude qualitative a été menée et elle a ciblé les femmes, les hommes et les agents de santé villageois, interrogés à travers des entretiens individuels et des discussions de groupe. Les femmes ont joué un rôle financier très important dans les dépenses de nutrition et de santé. Grâce à des activités génératrices de revenus, elles parviennent à contrôler leurs finances et ont donc un contrôle important sur les achats de nourriture, soit au profit de leurs enfants, soit pour l'amélioration de leur propre alimentation et même dans la prise de décision. Les connaissances acquises ont permis de comprendre l'importance de l'espacement des naissances et donc d'accepter la planification familiale. Les femmes bénéficient d'un solide soutien de la part des hommes, bien que la prise de décisions concernant la recherche de soins et la planification familiale ne dépende pas totalement des femmes. Nous avons également noté la mise en place d'un cadre d'échange entre mari et femme. Mots-clés : autonomisation, planification familiale, enfants, Kangaba.

\section{ABSTRACT:}

Economically empowered women are more engaged in their own health as well as that of their children. With this in mind, the NGO IAMANEH Switzerland, in collaboration with the NGO GAD, implemented a project to empower women in the Kangaba circle. We conducted this study three years after the start of the project to assess the impact of this project. It was carried out in August 2018 in the communes of Balan Bakama, Séléfougou and Maramandougou of the Kangaba circle. A qualitative study was conducted that targeted women, men and village health workers surveyed through one-to-one interviews and group discussions. Women played a very important financial role in spending on nutrition and health. Through income-generating activities, they manage to control their finances and therefore have an important control over food purchases, either for the benefit of their children or the improvement of their own diet and even in decision-making. Knowledge has helped to understand the importance of birth spacing and therefore to accept family planning. Women enjoyed strong support from men, although decision-making about care seeking and family planning was not totally dependent on women. We also noted the establishment of a framework of exchange between husband and wife.

Keywords: Empowerment, family planning, children, Kangaba.

\section{INTRODUCTION - ENONCE DU PROBLEME}

L'autonomisation et l'équité concernant les femmes est un droit humain fondamental d'une importance critique dans la réalisation des objectifs de développement, y compris la santé (1).

En plus d'être un objectif en soi, l'autonomisation des femmes est également considérée comme un moyen d'atteindre d'autres résultats de développement importants, tels que l'amélioration de l'état nutritionnel des enfants. Étant donné que les femmes sont souvent les principales gardiennes du ménage, les dynamiques intrafamiliales qui déterminent l'allocation des ressources et leur impact sur le bien-être des individus font de plus en plus l'objet d'analyse (2).

Les femmes chargées de porter et de nourrir leurs enfants, jouent de surcroît un rôle vital même s'il n'est souvent pas reconnu, au sein de la famille, de la collectivité et de la société (3).

A travers le monde, les femmes contribuent de manière significative à la survie économique de leur communauté et à la croissance de leur pays en accomplissant un large éventail d'emplois et d'activités dans des secteurs peu valorisés (travaux domestiques, économie informelle...). Alors que les femmes effectuent $66 \%$ du travail mondial et produisent $50 \%$ de la nourriture mondiale, elles ne perçoivent que $10 \%$ des revenus (4). Elles représentent $70 \%$ du 1,3 milliard de personnes qui vivent avec moins de 1 dollar par jour (4).

Au Mali, 3,6\% de salariées sont des femmes actives. La quasi-totalité des femmes actives occupées évoluent dans le secteur informel avec un pourcentage de $88 \%$ en 2007 (5). 
Les femmes économiquement autonomes sont plus engagées dans des activités génératrices de revenus et maitrisent leurs finances et ont donc un contrôle important sur les achats de produits alimentaires, soit au profit de leurs enfants ou à l'amélioration de leur propre régime alimentaire (6).

La nutrition est un pilier de base pour le développement social et économique des communautés et d'un pays. Les actions menées pour réduire la malnutrition et la mortalité chez les nourrissons et les jeunes enfants sont importantes pour atteindre les objectifs du développement durable (7).

Plus de 3 millions de décès d'enfants évitables chaque année est attribuable à la malnutrition (8).

La malnutrition pendant la petite enfance, affecte les fonctions vitales notamment cognitives et permet l'installation de la pauvreté à travers des obstacles liés à une faible capacité d'apprentissage et de production (7). Actuellement dans le monde, selon la Banque Mondiale en 2017, environ 151 millions d'enfants de moins de 5 ans souffrent de retard de croissance soit $22,2 \%$ et 51 millions d'enfants souffrent de malnutrition aigüe sévère soit 7,5\% (9).

En Afrique 58,7millons des enfants souffrent de retard de croissance et 13,8 millions de malnutrition aigüe sévère (9). La malnutrition constitue un problème majeur de santé publique en Afrique de l'ouest avec $29,9 \%$ de retard de croissance et $8,1 \%$ de malnutrition aigüe sévère chez les enfants de moins de 5 ans (9).

$\mathrm{Au}$ Mali, selon l'enquête SMART 2018, 11,2\% des enfants de moins de 5 ans souffrent de malnutrition aigüe et $18,1 \%$ de retard de croissance (10). Au Mali, selon I'UNICEF en 2013, 110 enfants de moins de 5 ans meurent chaque jour de causes liées à la malnutrition soit 5 enfants chaque heure (11). Les recherches sur la relation entre l'autonomisation des femmes et la nutrition, en particulier la nutrition de l'enfant, ne cessent d'augmenter (2).

Au Mali, il existe peu d'études sur l'autonomisation des femmes, celles-ci étant restées longtemps en marge des instances de prise de décisions (5).

\section{MATERIEL ET METHODES :}

L'étude était qualitative et s'est basé sur des discussions de groupes, entretien individuel avec des informateurs clés du projet bénéficiaires (femmes/hommes), relais communautaires, équipe de projet, les services de santé) Les données nécessaires ont été collectées afin de pouvoir (i)Apprécier la capacité de prise de décision des femmes en matière de santé de la reproduction et de la planification familiale, et leur capacité d'autonomisation par l'augmentation de leur revenu à travers leurs activités de maraichage,

(ii) Déterminer les changements de comportement les plus importants survenus en matière d'alimentation adéquate pour les femmes enceintes et allaitant, les nouveau-nés, les enfants, mais aussi en matière de santé de la reproduction et de la planification familiale moderne,

(iii) Apprécier le degré d'implication et le soutien des hommes à leurs femmes pour la promotion de la santé et le processus de prise de décision et d'autonomisation ;

(iv) les changements de comportement les plus importants survenus dans le processus de transformation genre

(v) Déterminer le niveau de connaissance des mères en termes de besoins nutritionnels des femmes enceintes, des femmes allaitant, des nouveau-nés et des enfants à travers une alimentation équilibrée et

(vi) Apprécier le niveau d'offre de services de qualité en matière de santé de la reproduction et de planification familiale centrée sur les méthodes modernes.

- Des entretiens individuels à l'aide d'un guide d'entretien semi-structuré avec

o Les responsables du projet et ses partenaires (équipe, maris pionniers, et animateurs du GAD, la coordinatrice régionale de IAMANEH Suisse, les services techniques de la santé et du développement social au niveau du cercle, les leaders villageois, les responsables des CSCOM, les relais communautaires);

- Les bénéficiaires locaux du projet (mères, les pères de famille, les femmes pratiquant le maraichage, les membres des ASACO; personnel du CSCOM,

Des Focus groupes prenant en compte les groupements de femmes et les hommes chefs de ménage ont été réalisés à raison de 4 villages par commune. Il a été réalisé 3 focus femmes par commune, 1 Focus Hommes et 1 focus maris pionniers, en prenant en compte les gros et les petits villages en termes de population. Cette approche avait l'avantage de ne pas occulter les biais qui peuvent exister entre gros et petits villages.

- L'observation directe non participante a été réalisée pour apprécier les différentes réalisations en matière de la pratique du maraichage, le mode d'alimentation et l'état général des enfants.

\section{Gestion et analyse des données}

Les entretiens et discussions ont été préparés dans un format normalisé comprenant les informations sociodémographiques, la transcription et la traduction des verbatim en français sur la base de l'enregistrement audio numérique. Les transcrits ont été révisés afin de faire une catégorisation et un codage, ce qui a permis une analyse plus aisée avec le postage des extraits de textes pertinents sous les thèmes, catégories et codes. La triangulation et l'intégration des informations pertinentes de toutes les sources ont été faites afin de dégager une synthèse de la convergence ou la complexité de chaque thème. Les transcriptions ont été codées ligne par ligne en utilisant des thèmes détaillés et sous-thèmes identifiés à priori comme étant pertinent pour l'étude, ainsi que l'utilisation des thèmes détaillés et sous-thèmes qui ont émergé à partir des données. 


\section{RESULTATS :}

A. Connaissance du projet GAD et interactions, pertinence du projet dans la zone

Il y'a une connaissance générale du projet dans la zone. La population a une bonne appréciation du projet et de ses interventions. Le projet est connu à travers ses animateurs qui mènent des activités de sensibilisation et à travers les maris pionniers mis en place dans le cadre du projet. En termes de collaboration avec le personnel des structures sanitaires, le projet apporte un soutien de renforcement de capacité dans tout ce qui est qualité des soins à travers un bon accueil des patients. II contribue également à améliorer la fréquentation des centres de santé par les populations à travers ses activités de sensibilisation sur la santé et nutrition de la femme et de l'enfant.

Les interventions de GAD nous apportent beaucoup d'appuis dans le sens de la CPN à travers les sensibilisations et il y'a de plus en plus d'hommes qui accompagnent leurs femmes en CPN et CPON. Ce qui rend le suivi plus facile et effectif (Entretien individuel DTC Manicoura)

Le projet est également connu pour la mise en place de structure communautaire d'épargne d'argent appelée «Tekereni » qui est beaucoup appréciée par l'ensemble des répondants homme ou femme, ainsi qu'à travers les activités de jardinage développées dans certains villages du projet.

Nous connaissons GAD à travers le "Tekereni " qui consiste à regrouper les femmes chaque semaine pour échanger et cotiser une somme d'argent que nous pourrons utiliser afin de générer des ressources. Nous faisons des prêts entre nous et nous remboursons avec intérêt. Par exemple celle qui emprunte 5000FCFA doit rembourser avec un intérêt de 500FCFA (FGD femmes, Sélofara)

L'ensemble de ces activités contribuent à améliorer la santé et la nutrition des femmes et des enfants dans les communautés d'intervention. Cette amélioration s'explique par le fait que les problèmes résolus par le projet tel que la faible fréquentation des centres de santé, la non disponibilité des légumes pour l'alimentation ou la non implication des hommes dans la santé des enfants et des femmes étaient de véritables problèmes cités par la plupart des répondants. Ce qui met en évidence toute la pertinence du projet et de ses interventions pour les populations concernées

Nos maris ne s'occupaient pas de notre santé ainsi que de celle de nos enfants. Avec le projet GAD, ils ont compris qu'ils doivent le faire et la plupart des hommes le fait dans notre communauté maintenant. D'ailleurs, ils nous accompagnent vite au CSCom dès les premiers signes de maladies et payent nos médicaments parce que avec leur présence, les agents de santé les explique l'importance des médicaments et comment mieux faire le traitement. Alors à la maison, même si on oublie de prendre les médicaments, ils sont là et nous le rappelle. Cela contribue à nous guérir plus vite (FGD femmes, Séléfougou)

\section{B. Les apports du projet à la population}

Les plus grands problèmes de santé touchant les femmes et les enfants étaient la malnutrition, la non fréquentation des centres de santé et les naissances trop rapprochées. Avec l'arrivée du projet ces problèmes sont en majeur partie résolus. Les gens ne fréquentaient pas les centres de santé à cause de la mauvaise qualité de l'accueil de la part des agents de santé. Aujourd'hui ce problème est résolu car ceux-ci se sont rendu compte du problème et ont reçu des formations les permettant de corriger cela.

Ici chez nous, on ne se fatiguait pas pour aller au centre de santé car les agents de santé n'étaient pas accueillants et nous n'avions pas les soins adéquats bien que le cout soit élevé. Nous préférions nous soigner à la maison ou aller à Sélingué si la maladie se complique. (FGD hommes Balan Mansala)

Certains agents de santé ont confié qu'ils ne savaient pas que certains de leurs comportements envers les patients concourraient à les éloigner du centre de santé. Chose qui est désormais corrigée. Concernant la santé et nutrition des enfants, les femmes ont appris comment préparer des aliments enrichis avec les produits locaux pour les enfants. Elles ont compris l'importance de l'allaitement maternel exclusif jusqu'à 6 mois même si certaines dépassent les 6 mois avant d'introduire des aliments de compléments.

J'ai appris à faire la différence entre un bon accueil et un mauvais accueil lors des formations faites par GAD. En réalité moi je ne savais pas que la qualité de l'accueil des patients était importante. Depuis que nous avons amélioré notre accueil les gens fréquentent plus le centre de santé. (Entretien individuel, sage-femme Balan Mansala)

Les femmes et même certains hommes arrivent à faire le lien entre l'espacement des naissances et la bonne santé de l'enfant d'où l'acceptation de la PF même s'il reste encore à faire à ce niveau-là.

Moi j'aimerai bien pouvoir faire une méthode de PF pour espacer mes naissances parce que je sais qu'une femme qui espace ses naissances donne des enfants plus en santé et la femme elle-même se porte bien. Mais j'ai peur de mon mari. II ne veut pas entendre parler de PF. (FGD femmes Figuiracoro)

Pour la santé des femmes, les hommes sont désormais impliqués et reconnaissent la valeur ajoutée d'accompagner sa femme au CSCom pour le suivi correct du traitement et même des CPN et CPON. Les hommes ont affirmé que certaines femmes prenaient 
l'argent pour la CPN mais n'y allaient jamais. De plus ils n'étaient pas informés de l'état de santé de leurs femmes donc ils ne pouvaient pas compatir convenablement ou suivre leurs traitements comme il se doit.

Ici, tous les hommes accompagnent désormais leurs femmes au centre de santé, ce qui ne se faisait pas avant. Nous pensions qu'il fallait juste donner de l'argent pour la consultation. Maintenant nous avons compris l'importance de les accompagner. Il y'a certaines femmes qui prenaient l'argent de la consultation mais n'y allaient pas ou ne prenaient pas leurs médicaments comme il le faut. Maintenant nous les accompagnons nous-même et les agents de santé nous expliquent ce qui est bien pour elles et ce qui ne l'est pas pour elles. (FGD hommes Fouh)

Avec l'implication des maris ce problème est résolu et ils sont informés de tous les problèmes de santé de leurs femmes, ce qui contribue à renforcer le soutien qu'ils leurs donnent. La santé des enfants et des femmes n'est plus une affaire des femmes mais de tous.

Un homme qui s'implique dans la santé de sa femme et de ses enfants a plus de chance de dépenser moins d'argent car tu sais ce qui est bien et ce qui ne l'est pas pour eux. (FGD hommes Manicoura)

A travers l'instauration de la communication entre homme et femme, le climat familial est plus convivial et les soutiens plus forts. Ce qui n'existait pas en milieu culturel malinké avant le projet. II fallait se cacher pour parler à sa femme car cela était mal perçu de s'adresser à sa femme directement.

Grace aux conseils que les maris pionniers et l'animatrice de GAD nous donnent, nos maris sont plus attentionnés envers nous et nous écoutent. Ils sortent moins maintenant et nous causons le soir autour d'un thé. Ce qui ne se faisait pas du tout avant. (FGD femmes Mambila)

Avec la mise en place des structures communautaires d'épargne appelée « Tekereni », le projet a réussi à créer un climat d'entente entre les femmes elles même d'abord mais aussi entre les femmes et leurs maris.

Avec les revenus du "Tékéréni", nos maris nous considèrent plus car ils savent que nous pouvons aussi les aider financièrement au besoin. De plus quand nous nous retrouvons chaque semaine c'est pour échanger entre nous de nos petits problèmes et parfois l'animatrice de $G A D$ nous aide à trouver les solutions à ces problèmes. C'est un cadre d'échange que nous apprécions beaucoup et surtout que l'animatrice est très patiente et nous écoute sans se fâche. (FGD femmes Mambila)

Les femmes arrivent à bénéficier de prêt dans la caisse d'épargne qui leur permet de faire le petit commerce ou l'élevage de petits ruminants ou même d'acheter des engrais ou herbicides pour leurs champs. Avec les revenus que ces prêts engendrent, les femmes arrivent à s'occuper d'elles même pour le paiement des créances, les soins de santé de la famille et même des petits trucs plaisants pour leurs maris. Cela aussi contribue à renforcer le climat de convivialité dans la famille et les femmes sont aussi écoutées par les maris.

Pour les villages ayant bénéficié de périmètre irrigué, c'est aussi un moyen de gain supplémentaire pour la famille. Ils consomment une partie et en vendent l'autre pour subvenir aux petits besoins de la famille y compris ceux du mari.

Maintenant nous nous entendons très bien avec nos maris et ils ont tous "la bouche dans l'huile". (Rires) Nous leur préparons des bons petits plats et du coup ils nous aiment plus et nous écoutent. Tout cela est possible grâce aux revenus que nous avons à partir de nos périmètres maraichers. Nous remboursons aussi nos petites créances. (FGD femmes Mambila)

Selon les femmes cette contribution financière les aide à consolider leur mariage et aussi à leur donner le droit d'être consultée par leurs maris pour les décisions concernant le foyer.

Et pour celles qui souhaitent faire la PF et qui n'ont pas le soutien du mari, elles peuvent se cacher pour aller le faire au CSCom avec les revenus qu'elles ont sans forcément passer par le mari.

Mon mari n'aime pas que je fasse la PF mais moi je le veux car je sais que ça me fait du bien ainsi qu'à mes enfants, donc avec l'argent que j'ai du maraichage je me cache pour aller au centre de santé et faire ma PF sans demander de l'argent à mon mari. (FGD femmes Sélofara)

Ces revenus constituent un véritable atout pour la considération des femmes dans la famille et pour la consolidation de leur foyer.

Certains hommes affirment avoir pris de l'argent à leurs femmes en prêt pour leurs besoins et cela montre le degré de considération de la femme par l'homme car il la sent utile et importante.

Les femmes aussi utilisent ces revenus pour plaire plus aux maris en leur préparant des petits plats.

\section{Nouvelles connaissances acquises et} difficultés pour les appliquer

Avant le projet aller au centre de santé était considéré comme une perte de temps et de ressource parce que selon les répondants ils n'avaient pas satisfaction quant aux services offerts dans ces structures de par la qualité de l'accueil mais aussi la qualité des soins de façon générale.

Les services de santé à travers les renforcements de capacité reçue ont amélioré la qualité des services et ont compris que la qualité de leur travail dépend de l'accueil qu'ils donnent aux patients. Cela a contribué à augmenter le niveau de fréquentation des centres de santé 
Les interventions du projet GAD contribue à augmenter mon taux de CPN parce que les femmes viennent de plus en plus au centre de santé surtout avec l'implication des maris. II faut aussi noter qu'après avoir participé à des formations de GAD j'ai compris qu'il fallait donner un bon accueil aux patients pour qu'ils viennent plus au centre de santé. (Entretien individuel sage-femme Balan Mansala)

Concernant la population générale, elle a compris toute l'importance d'aller faire les CPN durant la grossesse et aussi de l'espacement des naissances. Avant le projet, les hommes ne s'occupaient pas de la santé des femmes et des enfants. Le plus important pour eux était de donner l'argent qu'il faut pour aller en consultation et le reste à faire relavait uniquement de la femme. Le projet à travers les maris pionniers a pu mettre en évidence toute l'importance de l'implication des maris au-delà de l'aspect financier. Un mari qui accompagne sa femme au CSCom a plus de chance de mieux comprendre l'état de santé de sa femme, de mieux la soutenir et de veiller au suivi correct de son traitement.

Ainsi beaucoup d'hommes dans ces communautés accompagnent désormais leurs femmes et enfants et veillent au suivi des traitements parce qu'ils ont compris l'importance et ont vu les maris, pionniers le faire en exemple. Concernant l'espacement des naissances, les femmes ont entièrement compris l'importance même si la plupart ne décide pas d'elles même le fait d'aller faire une méthode de PF. Les hommes ont également compris, mais il y'a toujours à faire car certains sont toujours sur la défensive lorsqu'on leur parle de PF. Ils pensent que les méthodes de PF font plus de mal que de bien car rendant les femmes malades.

Moi j'ai compris que l'espacement des naissances est bien pour la femme et aussi pour l'enfant et donc pour l'homme aussi car il y'aura peu de maladies. Mais ce que je n'aime pas ce sont les effets secondaires engendrés par la PF. Il parait que ça fait plus de mal que de bien en rendant la femme maladive. (FGD hommes Manicoura)

Par ailleurs, la communication entre mari et femme est perçue comme une nouvelle connaissance assez constructive tant pour les femmes que pour les hommes. Cette pratique était très rare avant le projet. Maintenant ils ont compris que c'est très important et que ça les permet de mieux gérer leurs familles. Tous les répondants ont affirmé avoir des discussions de concertation avec leurs femmes depuis l'arrivée du projet et qu'ils sont convaincus de son intérêt.

Moi je ne pars plus souvent au " grin " parce que je reste causer avec ma femme. Avant nous ne faisions pas cela et les femmes ne se sentaient pas aimer. Donc elles nous rejetaient souvent au lit. Maintenant elles ne nous rejettent plus et tout va bien entre nous. Nous causons et nous partageons nos idées ensemble. Cela est un environnement familial plaisant pour les enfants également car en nous voyant causer ensemble, l'enfant se déplace entre nous et on peut sentir qu'il est très content. (FGD hommes Balan Mansala)

Les femmes aussi apprécient beaucoup le fait de pouvoir désormais passer du temps avec leurs maris en discutant et souvent autour d'un thé. Le cadre de vie familial est convivial avec ce type d'échange d'après la plupart des répondants.

II n'y'a pas de difficulté citée pour l'application de ces nouvelles connaissances de la part des hommes. Pour les femmes, la difficulté se situe au niveau de la PF. Même si elles ont compris l'importance et la nécessité de pratiquer la $\mathrm{PF}$, elles ne sont pas entièrement responsables de la prise de décision.

Parfois il est difficile pour nos maris d'accepter de faire la PF et s'ils ne sont pas d'accord nous on ne doit pas le faire. (FGD femmes Séléfougou)

Alors que certains maris sont encore réticents face à cela, il faudrait continuer les sensibilisations et amener ces hommes à accepter afin qu'ils puissent autoriser leurs femmes à appliquer une des méthodes de PF pour le bien-être de la famille.

\section{Perceptions sur les maris pionniers et les conseils promus par GAD}

Dans les communautés, les maris pionniers sont pris pour exemple et sont écoutés par les autres. Ils profitent de tous les rassemblements pour faire passer les messages de sensibilisation sur le rôle des maris dans la santé de la femme et des enfants. Ils parlent également de la PF et de l'espacement des naissances.

Nous avons vraiment appris avec les maris pionniers et ils ne se fâchent pas. Depuis que nous ne les écoutions pas jusqu'à ce que nous les écoutions maintenant ils ne se sont pas découragés. Ils profitent de tous les rassemblements pour nous parler de notre rôle dans la santé et nutrition de nos femmes et de nos enfants. (FGD hommes Fouh)

En plus de faire véhiculer ces messages, ils les appliquent eux même d'abord et encouragent les autres maris à faire autant. Les maris pionniers sont acceptés par toutes les communautés du projet et leur rôle est considéré comme important pour le développement des communautés car les conseils promus sont pris comme une lumière qui vient clarifier les visions et perceptions. Vraiment le travail des maris pionnier est comme une lumière sur nos vies, car nous acceptons de faire des choses qui n'étaient pas acceptées avant telles que voir un homme prendre son enfant et l'amener au centre de santé. C'était une affaire de femme. Maintenant nous le faisons et même mieux que les femmes (Rires) En plus de cela, il y'a aussi la question de l'espacement des naissances. Chez nous, avoir beaucoup d'enfants était considéré comme signe de richesse et nous n'acceptions pas de parler de PF. Maintenant nous comprenons mieux son importance et l'impact qu'elle peut avoir sur la santé de nos enfants et de nos femmes (FGD Hommes 
Mambila)

\section{E. Difficultés rencontrées pour le suivi des conseils}

Les principales difficultés rencontrées par les maris pionniers et les animateurs du GAD sont le fait qu'il s'agit de communautés conservatrices. Certains conseils en lien avec les pratiques traditionnelles tel que l'excision et le lévirat sont difficiles à aborder. Et même s'ils arrivent à les aborder, les conseils ont moins d'effet sur les comportements.

Pour l'espacement des naissances, toutes les femmes ont compris l'importance pour leur santé et pour la santé des enfants, mais elles n'ont malheureusement pas tout le pouvoir de décision pour la pratique. Ce sont les maris qui décident et certains parmi eux sont encore réticents car ils ont encore des réserves sur les méthodes de PF. Ces réserves ont besoin d'être résolues pour plus d'efficacité.

Je sais que si les naissances sont espacées la mère et les enfants seront en bonne santé et je vais moins dépenser mais je ne veux pas créer d'autres maladies avec ces méthodes de PF. II faut trouver des méthodes qui ne rendent pas nos femmes malades. (FGD hommes Manicoura)

\section{F. Existence de pratiques traditionnelles néfastes pour la santé des femmes et des filles}

Dans ce milieu culturel malinké les pratiques traditionnelles comme l'excision ou le lévirat existent encore fortement. Pour l'excision, la plupart des répondants ont préféré ne pas en parler car il s'agit selon eux d'une pratique traditionnelle ancienne pratiquée par leurs ancêtres et qu'ils préfèrent ne pas en parler.

L'excision est une tradition que nous avons trouvée et nous continuons à la pratiquer et d'ailleurs nous préférons ne pas en parler. (FGD femmes Kolondjigué)

Le lévirat est perçu comme une pratique traditionnelle positive et de solidarité. Pour les répondants si une femme après le décès de son mari est remariée par le frère de ce dernier cela signifie qu'elle est une bonne épouse. Et si elle n'est pas remariée par un frère du mari, c'est la honte et le désespoir car elle sera obligée d'aller se remarier dans une autre famille et avoir des enfants de noms de famille différents.

Ici, il faut qu'un frère de ton mari te prenne comme épouse après son décès sinon qui va s'occuper de tes enfants et en plus c'est un soutien que le frère de ton mari te donne en te remariant.

(FGD femmes Mambila)

G. Capacité de prise de décision des femmes en matière de santé de la reproduction et de la planification familiale, et leur capacité d'autonomisation par l'augmentation de leur revenu à travers leurs activités de maraichage

Par rapport à l'autonomisation des femmes, la plupart se sentent consultée pour les prises de décision concernant la gestion de la famille. Cependant, certaines signalent toujours que pour l'utilisation des revenus du maraichage, il faut qu'elles en discutent avec leurs maris. Ce qui montre que l'autonomisation est partielle et qu'il faudrait encore faire un peu plus dans ce sens.

Moi j'utilise mes revenus comme je le veux mais il faut d'abord que mon mari me donne son autorisation. (FGD femmes Séléfougou)

Cela s'explique par le fait qu'il s'agisse d'une communauté dans laquelle le pouvoir de décision appartenait uniquement au mari avant l'intervention du projet et cela n'est pas toujours facile à faire changer. II faut tout de même noter un grand changement dans le fait que les femmes ont désormais le droit de s'assoir avec leurs maris pour discuter et prendre ensemble les décisions.

Avec ma femme je partage mes idées et je fais attention aux conseils qu'elle me donne. Nous prenons ensemble les décisions. (FGD hommes Manicoura)

H. Connaissance des mères en termes de besoins nutritionnels des femmes enceintes, des femmes allaitant, des nouveau-nés et des enfants à travers une alimentation équilibrée

A ce niveau, les femmes ainsi que les hommes ont aisément compris la nécessité d'améliorer la qualité de l'alimentation de la femme enceinte et allaitant et même celui du nouveau-né et des enfants. Les femmes reçoivent de la viande et du poisson en plus durant la grossesse et l'allaitement et leurs maris sont aux petits soins, ce qui est très apprécié par l'ensemble des femmes

Les femmes ont également appris comment améliorer la qualité de l'alimentation des enfants à travers les produits locaux et la bouillie enrichie. Elles disent avoir appris cela à travers les messages de sensibilisation des animateurs de GAD lors des séances de démonstrations nutritionnelles. En plus des repas familiaux, la plupart des femmes prévoient un plat supplémentaire pour les enfants qu'ils appellent «Bolomasigui ». Donc les enfants ont accès à la nourriture à tout moment de la journée et cela même en l'absence de la mère. Le « Bolomasigui » est préparé et gardé à la portée des enfants qui peuvent s'en servir autant de fois qu'ils le souhaitent durant la journée.

L'animateur de GAD nous a appris comment préparer la bouillie enrichie pour nos enfants et il nous a aussi expliqué que nous devons 
manger plus d'aliments enrichies pendant la grossesse pour que le bébé se porte bien. Les autres enfants aussi doivent voir accès à la nourriture autant qu'ils en veulent. C'est pour cela qu'ici toutes les femmes préparent le "Bolomasigui » pour les enfants. Les mamans aussi profitent bien de ce "Bolomasigui » (FGD femmes Figuiracoro)

I. Niveau d'offre de services de qualité en matière de santé de la reproduction et de planification familiale centrée sur les méthodes modernes

Le plus grand problème cité par les répondants par rapport à l'offre de services de qualité, a été la qualité de l'accueil au niveau des structures sanitaires, mais aussi la non disponibilité de médicaments.

Les répondants affirment que ces

problèmes sont résolus pour ce qui

concerne la qualité de l'accueil dans les

structures sanitaires depuis l'arrivée du projet GAD. Ils témoignent que les agents de santé sont plus disponibles, accueillants et patients.

Depuis l'arrivée de GAD, nous sommes satisfaits de nos agents de santé car ils sont plus accueillant et s'occupent bien de nous. Quand nous allons pour l'accouchement maintenant, les agents de santé ne s'impatientent plus et ils supportent toutes nos caprices avec patience. Quand tu arrives avec ton enfant malade, ils courent pour le prendre et le soigner très rapidement. Vraiment maintenant nous ne pouvons rien les reprocher (FGD femmes Figuiracoro)

Cependant, le problème lié à la disponibilité des médicaments est toujours présent, soit par absence des médicaments soit à cause du cout élevé.

$\mathrm{Au}$ niveau des structures sanitaires, les agents pensent que le projet contribue à faciliter leur travail dans le sens ou les femmes font de plus en plus de CPN et CPON et que leurs maris également les accompagnent au CSCom. Cette implication des maris est un plus car le suivi des traitements et des rendez-vous est plus rigoureux avec eux.

Avec l'implication des hommes dans la CPN, les femmes prennent plus leurs médicaments et suivent les rendez-vous. Les hommes sont plus observant que les femmes donc leur implication nous aide énormément. (Entretien individuel sage-femme Balan Mansala)

II faut noter également la sollicitation des services de PF par les femmes et pour la plupart accompagnées par leurs maris ou confirmant que ceux-ci sont consentants malgré la réticence de certains encore. Dans l'ensemble les hommes ont compris l'importance de l'espacement des naissances en termes d'éviction de maladies et donc d'épargne de ressources, mais sont encore sous réserve quant à la pratique. Cela s'explique par le fait qu'il existe encore des préjugés sur les méthodes de PF
Une femme qui est enceinte chaque année n'aura finalement plus de force pour donner naissance à des enfants en bonne santé. Alors que si les naissances sont plus espacées, elle sera en bonne santé ainsi que son enfant et donc nous les maris nous aurons moins de dépenses à faire. C'est pour cela que j'accompagne ma femme pour faire la PF. Mais chez nous ici, certains hommes pensent que la PF est source de maladies chez la femme et ils s'en méfient (FGD hommes Balan Mansala)

\section{J. Observation des périmètres maraichers}

L'observation des périmètres maraichers a permis de déceler certains problèmes déjà énumérés par les répondants tels que l'insuffisance d'eau pour les cultures. Le projet a mis à disposition des puits à grands diamètres mais qui tarissent pendant la saison sèche. Les communautés ont-elles même creusé des puits à petits diamètre en appui mais qui tarissent également. La surface de chaque périmètre maraicher est d'environ un demi-hectare qui est exploité par un grand nombre de personnes, entre 35 et 49 personnes. Ce qui est très limite pour le soutien à chaque personne. L'observation ayant été faite pendant la saison des pluies, la plupart des gens avaient fait des cultures saisonnières collectives sur l'ensemble de la surface telles que le maïs. Ces cultures collectives seront vendues et les revenus partagés entre les membres. Par contre dans certains périmètres, on a pu observer une abondance en légumes (aubergines, poivrons, choux et piment) cultivées de façon séparées. Chacun cultive sa portion de terre selon ses préférences en légumes

\section{CONCLUSION :}

Les femmes jouaient un rôle financier très important dans les dépenses pour la nutrition et la santé et l'autonomisation financière des femmes a été un facteur déterminant à cela. L'implication des hommes dans les soins de la femme et de l'enfant a aussi été un atout de taille pour l'amélioration de la santé et la nutrition.

\section{REFERENCES}

1. Parténaires en population et développement. Promouvoir l'autonomisation des femmes pour améliorer la santé des femmes et des enfants. Pékin, chine: the 
partnership for maternel, Newbom et Child Health; 2013 oct p. 5.

2. Mara V den B, Agnes QR, Stuart G. Women's empowerment and nutrition. Int Food Policy Reaearch Inst. oct 2013;

3. COULIBALY D. Relation entre le statut nutritionnel des enfants de 6 à 59 mois et l'alimentation des mères dans le CSCOM de NIAMAKORO II. Bamako: université des Sciences, des Techniques et des Technologies de Bamako; 2015.

4. Maruani M. Travail et genre dans le monde. Paris: Découverte; 2013.

5. SYSTEME DES NATIONS UNIES AU MALI. Mise en œuvre des objectifs du millénaire pour le développemrnt au Mali. Mali; 2009.

6. Muzi NL, Larissa J, Sameera TA, Saifuddin A. Association between women's empowerment and infant and child feeding practices in sub-Saharan Africa: an analysis of Demographic and Health Surveys. Public Health Nutr. 2015;18(17):3155-65.

7. Institut Nationel de la Statistique. Enquête Nationale Nutritionnelle Anthropométrique et de Mortalité rétrospective suivant la méthodologie. 2017. Mali: PAM, OMS, UNICEF, FAO; 2017.

8. Cunninghan K, Ruel M, Ferguson E, Uauy R. Women's empowerment and child nutritional status in South Asia: a synthesis of the literature. 2015;11(1):19. 9. Hayashi C, Krasevec J, Kumapley R, Mehra V, Borghi E, Flores-Urrutia MC, et al. Levels and trends in child malnutrition. Banque mondiale, UNICEF, OMS; 2018 p. 16.

10. Institut Nationel de la Statistique, Ministère de la Santé. Enquête Nutritionnelle et de Mortalité Rétrospective suivant la méthodologie SMART au Mali. Mali; 2018 p. 30.

11. Ackermans F. Sécurité nutritionnelle et alimentaire au Mali : compréhension et réponse. Mali: UNICEF; 2013 avr.

12. AG Iknane A. Etude de base d'un projet de lutte contre la malnutrition infantile et maternelle dans les communes de selefougou et saramandougou sur la rive droite du niger et de balanbakama sur la rive gauche dans le cercle de kangaba. 2015. IAMANEH SchweizSuisse; 2015

13. Dartch EK, Acquah A, Kumi-Kyereme A. Correlates of stunting among children in Ghana. BMC Public Health. 2014;14:504.

14. OUMAR A. Profil nutritionnel et de consommation alimentaire de la femme enceinte et allaitant vue au Centre de Santé Communautaire de Yirimadio en commune VI. Université des Sciences, des Techniques et des Technologies de Bamako; 2017. 15. INFO-STAT, Cellule de Planification et de Statistiques (CPS), Ministère de la Santé Institut National de la Statistique (INSTAT), ICF International. Enquête démographique et de Santé du Mali. ICF International Calverton, Maryland, USA; 2013 mai. 